\title{
HOMOSEXUALIDAD, ¿QUÉ ACTITUD TIENEN LOS UNIVERSITARIOS AL RESPECTO?
}

Baena Pérez, Daniel; Calvente Pérez, María del Rocío; Díaz Sánchez, Marina. Universidad de Granada

\section{RESUMEN}

El trato hacia los homosexuales siempre ha despertado mucha controversia, especialmente ahora que ha aumentado el interés por este fenómeno debido a que ha dejado de ser un tema tabú, al menos en la cultura occidental. Múltiples factores socioculturales han influido en este cambio de visión, factores como el uso de internet como medio libre de expresión o los cambios en las condiciones socio políticas del país (Ahmad, 2010). Estos cambios afectan tanto a jóvenes como a adultos quienes se están adaptando a esta visión de la sexualidad. El objetivo de esta investigación es analizar la actitud hacia personas homosexuales para ver si estos cambios socioculturales han ayudado a estrechar la diferencia entre hombres y mujeres (Cárdenas y Barrientos, 2008) o si por el contrario sigue presente, en cuyo caso será necesario crear nuevos planes de intervención que distingan entre hombres y mujeres.

Palabras clave: Homofobia, prejuicio, adaptación social, cambio de actitud

El 17 de mayo de 1990 marca un día histórico en la lucha contra la homofobia ya que fue en esta fecha cuando la Organización Mundial de la Salud retiró la homosexualidad de la lista de enfermedades mentales (OMS, 1990). 23 años más tarde, a pesar de lo que se ha luchado por erradicar esta discriminación ilógica e irracional, todavía queda mucho para que las parejas homosexuales puedan vivir con los mismos derechos sociales que las parejas heterosexuales y tengan las mismas oportunidades.

Las personas homosexuales son un grupo minoritario, lo que por desgracia hace que todo sea más novedoso, que sobresalga más y se vea como discordante. Esto hace que se produzcan ataques hacia ellos ya sea de manera directa en forma de agresiones físicas o un tipo de agresión más sutil en la que se incluirían aquellas conductas de discriminación hacia el colectivo que tienen como fin último marcarlos como un grupo social con menos derechos que el resto. Las conductas homófobas cumplen ciertas funciones para la persona que las realiza dotándolas de sentido y fomentando, además, que esta conducta se mantenga a lo largo del tiempo como ya propuso Katz (citado por Muchinik \& Seidmann, 1983) en su obra, dichas serían las siguientes:

Una función de adaptación en el sujeto el cual busca pertenecer a un grupo dentro de la sociedad, la pertenencia de una persona homosexual a este grupo perturbaría al sujeto lo que puede desembocar en conductas de evitación.

Una función de defensa del yo mediante la cual el sujeto expresa sus valores, en nuestro caso en particular puede usarse como un mecanismo de defensa. La persona por el mero hecho de ser hombre o mujer tiene unas ideas preconcebidas de cómo debe comportarse, un modelo a seguir. Nunca se plantearía la relación con personas del mismo sexo 
Una función de expresión de valores donde el sujeto expresa el tipo de persona que quiere llegar a ser, en nuestro caso, si el sujeto muestra una actitud positiva hacia la homosexualidad esto podría significar que quieren serlo y provoca rechazo.

Y una función de conocimiento que ayuda al sujeto a obtener una representación simplificada de la realidad que le rodea, la homofobia en este sentido permitiría una evaluación rápida, genérica y en la mayoría de los casos negativa, de todas las personas que formen parte de este colectivo Además de cumplir dichas funciones, consideramos una conducta como la manifestación de una actitud. Allport (1962) define la actitud como "Una disposición mental y neurológica, que se organiza a partir de la experiencia que ejerce una influencia directriz o dinámica sobre las reacciones del individuo respecto de todos los objetos y a todas las situaciones que les corresponden".

Krech, Crutchfield y Ballechey (1978) entran en más detalle definiendo la actitud como "una organización permanente de procesos emocionales, conceptuales y cognitivos con respecto a algún aspecto del mundo del individuo" lo que ha llevado a dividir esta en tres componentes: cognitivo, conductual y emocional. Según esta división la homofobia seria el componente emocional.

A su vez, Allport (1962) define al prejuicio como "una antipatía que se apoya en una generalización imperfecta e inflexible. Puede sentirse o expresarse. Puede estar dirigida hacia un grupo en general, o hacia un individuo por el hecho de ser miembro de ese grupo. El efecto final del prejuicio es colocar al objeto del mismo en una situación de desventaja no merecida por su propia conducta".

La mayor parte de las veces el prejuicio es negativo y acostumbra a incluir sentimientos de desprecio y desagrado hacia la persona o grupo al cual es dirigido. Billing (1975) define, en concordancia con Allport (1962), al prejuicio como un juicio previo, a priori, sin existencia de pruebas, como opiniones dogmáticas y desfavorables respecto a ciertos grupos y, por extensión, respecto a los miembros individuales que pertenecen a dicho grupo. Billing (1975) comprende al prejuicio como una actitud negativa y lo diferencia de la discriminación, que es comprendida por él como el prejuicio hecho conducta, es decir, la actitud sería el prejuicio y la discriminación sería la conducta.

Pero actualmente los cambios socioculturales mencionados anteriormente han dado lugar a la presencia de fuertes presiones normativas en favor de la igualdad y la tolerancia, haciendo que el prejuicio en lugar de desaparecer se haya adaptado a los tiempos transformándose en algo más sutil. En consecuencia actualmente se diferencia entre prejuicio sutil y manifiesto (Pettigrew y Meertens, 1995)

A toda conducta discriminatoria, le subyace un prejuicio, pero no todo prejuicio se transforma en conducta. Sin embargo, Allport (1962) afirma que toda actitud negativa tiende, de algún modo, a expresarse en acción en cierto momento. Plantea distintos grados en la acción negativa, desde la menos enérgica hasta la más enérgica:

- Hablar mal: Los sujetos efectúan comentarios con otras personas que no pertenecen a la minoría.

- Evitar el contacto: Intentan por todos los medios no acercarse o interactuar con sujetos de la minoría.

- Discriminación: No se les permite el acceso a ciertos grupos o instituciones.

- Ataque físico: El prejuicio llevado a actos de violencia física o psicológica.

- Exterminación: Linchamientos y matanzas. 
Aunque esta definición de prejuicio nos sirve como base para elaborar nuestra definición semántica del constructo, que no será otro que el prejuicio hacia la población homosexual, es necesario adaptarlo a nuestros tiempos y a nuestro tema de estudio en particular. Por ello, decidimos eliminar el apartado referente a matanzas y linchamientos y añadir nuevos indicadores que midan el prejuicio sutil. De esta forma nuestra definición semántica estará formada por los siguientes componentes:

- Realizar comentarios negativos o de desprecio sobre personas homosexuales

- Considerar a las personas homosexuales como inferiores y con menos derecho que los heterosexuales

- Evitar el contacto físico o intentar por todos los medios no acercarse/ interactuar con personas homosexuales

- Abstenerse de ir a lugares donde pueda haber personas homosexuales

- Sentir malestar fisiológico o cognitivo en presencia de personas homosexuales

- Discriminarles ante el acceso a ciertas actividades, grupos o instituciones

- Agredir o sentir el impulso de atacar a personas homosexuales de forma física o psicológica

- Sentir malestar ante pensamientos de atracción sexual hacia personas del mismo género o que una persona homosexual se sintiera atraída por la persona en cuestión.

Pero tal vez lo más interesante es que este prejuicio hacia personas homosexuales no se manifiesta de manera equitativa en hombres y mujeres, mostrándose estas últimas más tolerantes al respecto como ya nos informa la investigación llevada a cabo por Cárdenas y Barrientos con población universitaria (Cárdenas y Barrientos, 2008). Sin embargo estas investigaciones se realizaron acorde a la definición semántica de constructo anterior por lo que queremos comprobar si estas diferencias seguirán presentes al usar nuestra definición del constructo en la que se han contemplado aspectos más actuales.

Por tanto el objetivo de esta investigación es comprobar si existen diferencias de género respecto a la actitud hacia los homosexuales en la población universitaria. Para ello se evaluó la actitud hacia los homosexuales y se compararon las posibles diferencias entre hombres y mujeres controlando, además, variables como el tener personas homosexuales en el entorno con el objetivo de evaluar su posible influencia.

\section{MÉTODO}

\section{Participantes}

Se recogieron respuestas de 195 sujetos seleccionados al azar con edades comprendidas entre los 18 y los 30 años. De ellos, 55 fueron descartados por su condición sexual, es decir, si eran homosexuales o bisexuales ya que esto hubiera sesgado nuestra investigación. De los 140 restantes, 74 fueron mujeres y 66 fueron hombres, con una media de edad de 21'1 y desviación típica de 2'01. Todos ellos cursaban estudios universitarios.

\section{Instrumento}

Aplicamos la escala traducida del "Index of attitudes Toward homosexuals" (Hudson y Ricketts, 1980) cuyo objetivo original era medir la actitud hacia personas homosexuales acorde a la definición de homofobia de Weinberg (1972)La escala 
original cuenta con 25 ítems a ser respondidos por los sujetos mediante una escala tipo Likert de 1 a 5 (ver anexo 1).

La fiabilidad de la escala original, calculada mediante el coeficiente alfa de Cronbach, obtuvo una puntuación de $\alpha=0.90$ (Hudson y Ricketts, 1980). El alfa de Cronbach "de la IAH traducida al español fue de $\alpha=0.89$ (Caleb, 2005), lo que llevó al autor a concluir que con la traducción "no amenazo la validez construida por los autores".Para el cálculo de puntuaciones se invirtieron cierto número de ítems debido a su connotación negativa, es decir, donde el sujeto puntúa 1 se transforma en 5 , donde puntúa $2=4$, donde $4=2$ y donde $5=1$. La puntuación $=3$ se mantiene $\sin$ cambios. estos ítems son los siguientes: 3, 4, 6, 9, 10, 12, 13, 14, 15, 17, 19, 21 y 24 (anexo 1). Estos ítems se puntúan a la inversa porque son afirmaciones negativas, y si el sujeto responde la opción "1" de la escala Likert (totalmente de acuerdo) demuestra homofobia, entonces, se invierte la puntuación para mantener la idea inicial de los autores: "a mayor puntuación, mayor nivel de homofobia".

Dado que nuestro constructo incluye nuevos elementos realizamos un breve estudio para comprobar la validez de contenido. En dicho estudio le pedimos a un total de cinco expertos en la materia que valoraran el grado en que cada ítem representaba el constructo. Esta valoración se llevó a cabo mediante una escala Likert con tres alternativas de respuesta según el grado de acuerdo o desacuerdo con la asociación.

La valoración de los expertos nos demostró que los aspectos 1 y 7 de nuestra definición semántica no tenían ningún ítem asociado en la escala original por lo que decidimos añadir 5 ítems adicionales con el mismo formato de respuesta tipo Likert (ver anexo 2). También nos sugirieron eliminar el ítem número 11 de la escala traducida (Me sentiría cómodo/a sabiendo que mi sacerdote es homosexual) ya que la población universitaria actual es predominantemente no creyente como ya comprobaron España et al. (2001)

\section{Procedimiento}

En primer lugar, la escala traducida del "Index of attitudes Toward homosexuals" (Caleb, 2005), fue evaluada por un grupo de expertos que valoraron si los ítems estaban relacionados con los indicadores de la definición semántica que queríamos medir.

Una vez que los expertos aprobaron los ítems finales, adaptamos nuestro cuestionario a la aplicación electrónica LimeSurvey, un servicio de encuestas online que nos permitió aplicarlo vía Internet. La distribución del cuestionario se realizó por medio de cadena de correos electrónicos a estudiantes universitarios de carreras variadas y mediante la distribución de códigos QR impresos (ver anexo 3). Decidimos que este era el método más cómodo por ser rápido, fácil y sin limitaciones horarias, además aseguraba un nivel de anonimato mayor del que se hubiera conseguido pasándolo en persona.

Una vez que el participante accedía al enlace, en una página de bienvenida se le daban instrucciones y se le advertía del anonimato de la encuesta. Tras esto, se pasaba a la primera parte del cuestionario donde se registraban los datos sociodemográficos y por último se presentaban los 30 ítems de la escala, los cuales se contestaban mediante una escala tipo Likert con valores de 1 a 5 , siendo 1 totalmente en desacuerdo y 5 totalmente de acuerdo. Una vez finalizado, los resultados se añadían automáticamente a una base de datos que posteriormente exportamos al 
programa "Statistical Package for Social Sciences" (SPSS versión 21.0), con el cual realizamos los análisis estadísticos pertinentes.

\section{RESULTADOS}

Con el objetivo de agrupar a los participantes según sus puntuaciones creamos los siguientes grupos en base a la puntuación total obtenida: Grupo de baja tolerancia (30100), grupo de tolerancia media (101-125) y grupo de alta tolerancia (126-150). Una vez sumadas las puntuaciones obtenidas de cada participante se le asigna a uno de los tres grupos previamente descritos. En la Tabla 1 y Figura 1 se refleja dicha asignación así como las diferencias entre hombres y mujeres que en este caso fueron significativas (chi-cuadrado $<0,005$ ).

Tabla 1. Distribución en grupos según sexo y puntuación total obtenida

\begin{tabular}{|l|l|l|l|l|}
\hline Sexo & Baja tolerancia & $\begin{array}{l}\text { Tolerancia } \\
\text { media }\end{array}$ & Alta tolerancia & $\%$ \\
\hline Femenino & 0 & 25 & 49 & $52,86 \%$ \\
\hline Masculino & 11 & 27 & 28 & $47,14 \%$ \\
\hline$\%$ & F 0\% & F 33,78\% & F 16,21\% & \\
& M 16,6\% & M 40\% & M 42,42\% & \\
\hline
\end{tabular}

Figura 1. Distribución en grupos según sexo y puntuación total obtenida

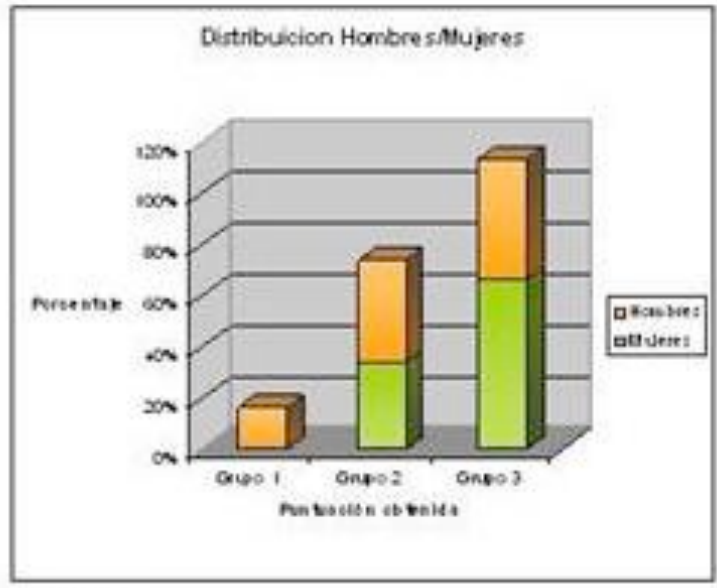

Figura 1.

Como muestra la Tabla 1 , en el grupo de baja tolerancia no hay ninguna mujer a diferencia de los hombres estando presentes en un 16'6\%. En el grupo de tolerancia media hay un $3778 \%$ de mujeres y un $40 \%$ de hombres, siendo este el grupo más igualado. $Y$ en el grupo de alta tolerancia hay casi el doble de mujeres que hombres.

Sumando las categorías que reflejan ausencia (grupos 2 y 3 ) y presencia (grupo 1) de niveles de homofobia se presentan los siguientes resultados: El 92'14\% de la muestra presentó un nivel bajo de homofobia y el $7^{\prime} 86 \%$ de la muestra presentó un nivel alto de homofobia estando este último grupo constituido exclusivamente por hombres.

En relación a las posibles diferencias entre participantes en función de la presencia de en su entorno, obtuvimos los siguientes resultados. 
Figura 2. Número de participantes con conocidos homosexuales en el entorno

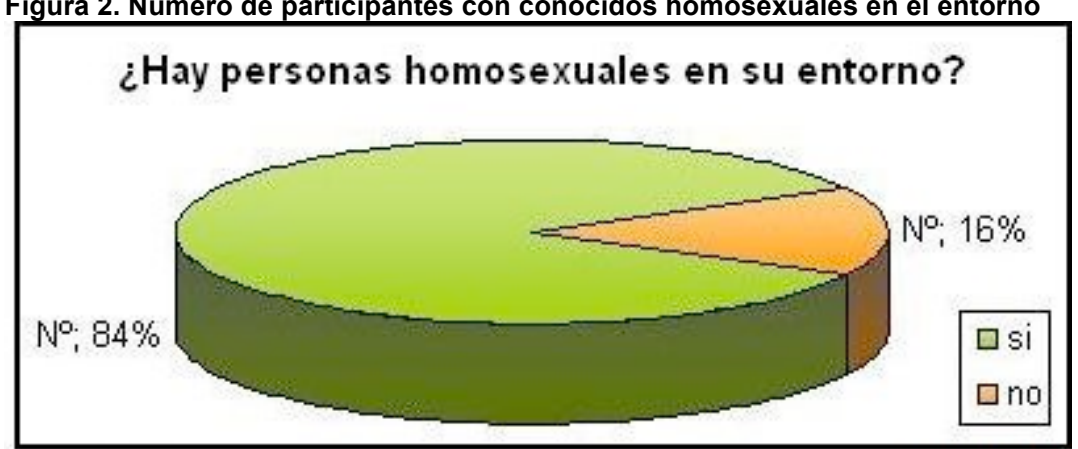

Como muestra la Figura 2, al haber tanta diferencia entre participantes con y sin personas homosexuales conocidas en el entorno, decidimos realizar el análisis con una submuestra compuesta por los 22 sujetos sin personas conocidas y otras 22 personas con conocidos elegidos al azar. Una vez obtenida la puntuación asignamos cada participante a uno de los tres grupos posibles, grupo de baja tolerancia (30-100), grupo de tolerancia media (101-125) y grupo de alta tolerancia (126-150). Los resultados de dicho subanálisis quedan recogidos en la tabla 2 .

Tabla 2. Distribución en grupos según conocidos en el entorno y puntuación total

\begin{tabular}{lllll}
\hline $\begin{array}{l}\text { ¿Hay personas } \\
\text { homosexuales } \\
\text { en su entorno? }\end{array}$ & Baja tolerancia & $\begin{array}{l}\text { Tolerancia } \\
\text { media }\end{array}$ & Alta tolerancia & Total \\
\hline Si & 2 & 7 & 13 & 22 \\
No & 3 & 13 & 6 & 22 \\
Total & 5 & 20 & 19 & 44 \\
\hline
\end{tabular}

Al realizar las pruebas de chi-cuadrado obtuvimos un valor de 0,101 lo que nos indica que las diferencias observadas no son significativas $(0,101>0,005)$ quedando descartada así nuestra segunda hipótesis según la cual el tener personas homosexuales en el entorno favorecería a tener una actitud más positiva hacia ellos. Por último, se realizó un análisis de fiabilidad del instrumento con el fin de comprobar la posible influencia de los ítems añadidos. Obtenemos evidencias de la fiabilidad del instrumento con un valor alfa de Cronbach de $\alpha=0,922$. Valor mayor que los obtenidos por la escala original y por la escala traducida. A pesar de esto tras un análisis más exhaustivo de los ítems añadidos observamos que si eliminamos los 4 últimos (ítems del 27 al 30) el coeficiente alfa seria aún mayor. Este hecho refleja que el aumento de longitud de la escala podría ser el factor responsable del aumento de fiabilidad.

\section{DISCUSIÓN Y CONCLUSIONES}

Respecto a nuestra primera hipótesis, el sexo femenino es más tolerante en el trato hacia la población homosexualidad, se ve confirmada, pues en el grupo de baja tolerancia no había presencia de ninguna mujer además de que en los grupos de tolerancia media y alta tolerancia obtuvieron mayores puntuaciones respecto a los hombres. La segunda hipótesis, tener en el ambiente cercano personas homosexuales hará que la persona sea más tolerante, queda rechazada.

Los resultados del estudio manifiestan que la muestra seleccionada presenta bajos niveles de homofobia. Tal como se comentó al principio, la homofobia representa al componente afectivo del prejuicio en lo referido al comportamiento respecto a los 
homosexuales, es decir, los sujetos tienden a actuar de un modo positivo hacia los sujetos homosexuales y en lo referido a lo cognitivo, los sujetos no tendrían una imagen negativa del grupo homosexual.

La media de la muestra fue muy superior a la de la muestra de la investigación realizada por los creadores de la escala (Hudson, y Ricketts, 1980). Lo interesante de esto es que a pesar de que pasaron, entre una aplicación y otra de la escala 30 años, la fiabilidad del test sigue siendo la misma. Esto hace pensar que las actitudes aprendidas culturalmente han cambiado con el paso del tiempo. La sociedad ha comenzado a realizar un cambio de actitud en torno a las personas homosexuales. Sin embargo es necesario mencionar la diferencia de resultados entre hombres y mujeres, lo que hace pensar que las mujeres son más tolerantes respecto a la introducción de los homosexuales en el panorama social. La mujer siempre ha presentado características de comprensión y sensibilidad respecto a la imagen de hombría del sexo masculino, quizás la homosexualidad para el hombre es un ataque a su rol social pues los hombres en presencia de hombres homosexuales se muestran más incómodos.

En cuanto a la conducta manifiesta de la homofobia presentada por la muestra pareciera que los sujetos optarían por alguno de los dos primeros grados propuestos por Allport (1962) en la definición semántica: hablar mal o evitar el contacto. Dado el bajo nivel de homofobia presentado por la muestra muy pocos sujetos optarían por la discriminación o el ataque físico y si alguno lo hiciera probablemente sería un hombre ya que son los sujetos que presentaron mayores niveles de homofobia.

En conclusión, a pesar de los grandes avances sociales, aún queda un largo camino por recorrer. Se deben inculcar valores de tolerancia y respeto para que llegue a existir una comunidad donde los homosexuales sean uno más del colectivo social, consiguiéndose así una sociedad en igualdad de derechos y oportunidades. No podemos olvidar que toda nuestra muestra de sujetos eran estudiantes universitarios por lo que el nivel cultural es más alto, factor que ayuda a mantener una actitud más tolerante en general y que explicaría nuestros resultados. La investigación futura tendrá que contemplar, además de este factor, la evolución de la homofobia hacia un tipo de prejuicio más sutil.

\section{REFERENCIAS}

Ahmad, S., y Bhugra, D. (2010). Homophobia: An updated review of the literature. sexual and relationship therapy, 25 (4), 447-455.

Allport, G. (1997). La naturaleza del prejuicio. Buenos Aires: Editorial Universitaria de Buenos Aires.

Billing, M. (1975). Racismo, prejuicios y discriminación: En Moscovici, S. (1980). Manual de Psicología Social. Capítulo 17, Tomo II. Buenos Aires: Amorrortu.

Caleb, J. (2005). Réplica de una escala de medición de actitudes hacia la homosexualidad. (Tesis inédita de doctorado). Facultad de Humanidades. Universidad de Belgrano. 
Cárdenas, M. y Barrientos, J. (2008). Actitudes explícitas e implícitas hacia los hombres homosexuales en una muestra de estudiantes universitarios en Chile. Psykhe, 17 (2), 17-25.

España, A., Guerrero, A., Farré, J.M., Canella-Soler, J., y Abós, R. (2001). La homofobia en el medio universitario. Un estudio empírico. Cuadernos de medicina psicosomática y psiquiatría de enlace, 57, 41-55.

Fiske, S., (1998). Stereotyping, Prejudice, and Discrimination. En Gilbert, D., Fiske, S., Lindzey, G. (Eds.), Handbook of psychology (pp. 357-392). Boston: McGraw-Hill.

IBM Corp. Released 2012. IBM SPSS Statistics for Windows, Version 21.0. Armonk, NY: IBM Corp.

Kawakami, K., Dunn, E., Karmali, F., y Dovidio, J. F. (2009). Mispredicting affective and behavioral responses to racism. Science, 323, 276-279.

Meertens, R.W., \& Pettigrew, T.F. (1997). Is subtle prejudice really prejudice? Public Opinion Quarterly, 61, 54-71

Muchinik, E. y Seidmann, S. (1983). Actitudes y Cambio Social. Buenos Aires. Departamento de Publicaciones, Facultad de Psicología de la Universidad de Buenos Aires.

Nadal, K. L. (2013). That's so gay!: microaggressions and the lesbian, gay, bisexual, and transgender community. Washington, DC: American Psychological Association.

Pratte, T. (1986 and 1991). A Comparative Study of Attitudes Toward Homosexuality. Journal of Homosexuality, 26 (1), 77 - 88.

Pettigrew, T.F., \& Meertens, R. (2001). In defense of the subtle prejudice concept: a report. European Journal of Social Psychology, 31, 299-309

Pettigrew, T.F., \& Meertens, R.W. (1995). Subtle and blatant prejudice in Western Europe. European Journal of Social Psychology, 25, 57-75.

Shelton, K., y Delgado-Romero, E. A. (2011). Sexual orientation microaggressions: The experience of lesbian, gay, bisexual, and queer clients in psychotherapy. Journal of Counseling Psychology, 58, 210-221. 